\title{
Shape optimization of curves and surfaces considering fairness metrics and elastic stiffness
}

\author{
M. Ohsaki, T. Ogawa and R. Tateishi
}

\section{Struct Multidisc Optim (2003) 24:449-456}

Due to a most unfortunate misunderstanding an incorrect version of the following article was published in Struct Multidisc Optim 24, pp. 449-456. The final version of this article is reproduced below.

\begin{abstract}
A method is presented for generating round curves and surfaces allowing discontinuities in tangent vectors and curvatures. The distance of the center of curvature from the specified point is used for formulating the objective function that is a continuous function of the design variables through convex and concave shapes. It is shown that a shell with and without ribs can be generated within the same problem formulation if the minimization problem is converted into a maximization problem and the parameter region where integration is to be carried out is restricted in view of the sign of the curvature. Optimal shapes are also found under constraints on the compliance against static loads. A multiobjective optimization problem is solved by the constraint method to generate a trade-off design between roundness and mechanical performance.
\end{abstract}

Key words fairness metric, parametric surface, ribbed shell, roundness, shape optimization

\section{1}

\section{Introduction}

Fairness metrics have been extensively used for automatic generation of curves and for interpolation of points

The online version of the original article can be found at: http://dx.doi.org/10.1007/s00158-002-0258-3

Published online: 4 May 2004

(C) Springer-Verlag 2004

M. Ohsaki ${ }^{1,}$, T. Ogawa ${ }^{2}$ and R. Tateishi ${ }^{2}$

${ }^{1}$ Department of Architecture and Architectural Systems, Kyoto University, Sakyo, Kyoto 606-8501, Japan

e-mail: ohsaki@archi.kyoto-u.ac.jp

${ }^{2}$ Department of Architecture and Building Engineering, Tokyo Institute of Technology, Ohokayama, Meguro, Tokyo 152-8552, Japan by a smooth curve. In the conventional approach, the square norms of curvature and variation of curvature are minimized, respectively, to obtain the minimum energy curve and the minimum variation curve (Meier and Nowacki 1987; Moreton and Séquin 1992). Subramainan and Suchithran (1999) presented a method for adjusting the knot vector of a B-spline curve based on the derivative of curvature in the process of designing ship hulls. For surfaces, the principal curvatures, mean curvature, Gaussian curvature, and their derivatives can be used for formulating the fairness metrics (Hangen et al. 1995; Barnhill 1994; Sarraga 1998; Greiner 1994).

After fairness or smoothness is defined, an optimization technique is to be applied to obtain an optimal curve or surface that minimizes or maximizes the given fairness metric as objective function. Evaluation of the derivatives of curvatures, however, needs much computational cost especially for surfaces. Therefore, the fairness metrics involving differentiation of curvatures are not practically acceptable for large and complex structures.

In addition to the conventional fairness metrics based on the curvatures, several advanced formulations have been presented for designing smooth curves and surfaces (Rando and Roulier 1991; Roulier and Rando 1994). The fairness metrics are classified into roundness, rolling, flattening, etc. There have been several practical applications of those metrics to ship hull design (Nowacki and Reese 1983). Ohsaki and Hayashi (2000) presented a modified version of the roundness metric by Rando and Rourier for optimizing ribbed shells. It has been pointed out, however, that the fairness metrics by Rando and Rourier do not always conform to the human impressions (Ohsaki and Hayashi 2000; Gerostathis et al. 1999).

In most of the surface design methods, the surfaces are divided into several regions, each of which is defined by a parametric surface such as Bézier patch and B-spline patch. In this case, constraints should be given for continuity and intersection between the adjacent regions (Du and Schmitt 1990; Barnhill 1994). Boundary conditions should also be given to formulate a constrained optimization problem (Nowacki et al. 1990; Welch and Witkin 
1992). The formulations for continuity in curvatures of surfaces, however, are very complicated, and it is inconvenient that the constraints should be modified and optimization problem itself should be reformulated depending on the types of the desired surfaces; e.g., sometimes discontinuity is allowed between the tangent vectors in the adjacent regions.

Optimization of curved structures such as arches and shells under mechanical constraints is called shape optimization which has been extensively studied in the literature (Rozvany 1992). However, shape and topology optimization based on the ground structure approach with fixed nodal locations of the finite element model is out of scope of the present paper. We only consider optimization of curves and surfaces that represent arches and thin shells. Parametric surfaces such as Bézier surfaces and B-spline surfaces are very useful for generating a smooth surface within small number of design variables. Ramm (1992) optimized a shell defined by the Bézier surface considering stress deviation or fundamental frequency. The method has been extended to the problem with buckling constraints (Ramm et al. 1993) and optimization of membrane fabric structures (Bletzinger 1999). Ohsaki et al. (1998) presented a trade-off design method between smoothness and elastic stiffness of an arch-type truss. Kegl and Antes (1998) optimized a single-layer truss under constraints on stresses considering geometrical nonlinearity. Ohsaki and Hayashi (2000) presented a method for generating round ribbed shells. In their method, however, the number of ribs should be defined in advance, and the shell should be modeled by different number of Bézier surfaces depending on the number of ribs. It is not convenient that the problem formulation depends on the desired optimal shape.

In this paper, a method is presented for generating round curves and surfaces allowing discontinuities in tangent vectors and curvatures. The distance of the center of curvature from the specified point is used for formulating the objective function which is a continuous function of the design variables through convex and concave shapes. Since the derivatives of curvatures are not used, a ribbed shell can be generated without any trouble by specifying the center of curvature of the surface or the isoparametric curves. Optimal shapes are also found under constraints on compliance that is regarded as a mechanical performance measure. A multiobjective optimization problem is solved by the constraint approach to generate a trade-off design between roundness and mechanical performance.

\section{2}

\section{Shape optimization of plane curves}

\section{1}

\section{Problem formulation}

Let $\mathbf{x}(t)$ denote a plane curve defined by a parameter $t \in[0,1]$. The fundamentals on geometry of curves may be referred to; e.g., Faux and Pratt (1979), do Carmo (1976). Let $\mathbf{r}(t)$ denote the unit tangent vector of $\mathbf{x}(t)$. The unit normal vector $\mathbf{n}(t)$ is defined as shown in Fig. 1 by rotating $\mathbf{r}(t)$ by $\pi / 2$. The curvature of $\mathbf{x}(t)$ is denoted by $\kappa(t)$. The curvature of the curve shown in Fig. 1 has negative values, because the center of curvature exists in the opposite direction of $\mathbf{n}(t)$ from a point along the curve.

The curvature is an intrinsic property of a curve that does not depend on parameterization. Therefore, the fairness of a curve can be controlled through $\kappa(t)$. In this section, we present an optimization method for generating round curves for the given span length. A simple approach may be to specify the desired value $\bar{\kappa}$ of $\kappa(t)$ and minimize the norm of deviation $(\kappa(t)-\bar{\kappa})^{2}$. Moreton and Séquin (1993) noted, however, that a metric defined simply by $\kappa(t)$ or its derivative with respect to $t$ is scale variant; i.e., it depends on the size of the curve, and the tangent vectors should be given at the boundaries to obtain a desired shape by using such a metric.

For a single arch spanning two supports, the fundamental theorem of the local theory of curves guarantees that the arch is uniquely determined by specifying $\kappa(t)$ (do Carmo 1976). For double arches as shown in Fig. 2, they cannot be uniquely determined from $\kappa(t)$; i.e., the tangent vectors at two ends should be given. It is inconvenient, however, to specify tangent vectors especially if extended to surfaces. Therefore, we use the center of curvature for generating round curves and surfaces without assigning tangent vectors.

In a standard approach to generating a double arch as shown in Fig. 2, the total curve is divided into two regions, each of which is defined by a parametric curve. In this case, however, the problem formulation depends on the number of regions. If a sufficiently large number of regions are given, then constraints should be assigned for continuity between the adjacent regions to generate an optimal curve with smaller number of arches. The number of variables becomes unnecessarily large and the continuity

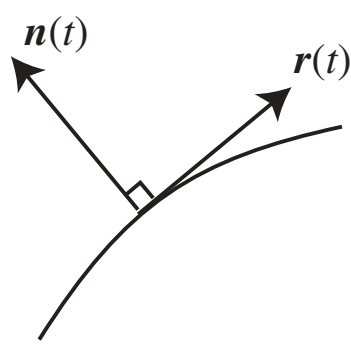

Fig. 1 Unit tangent and normal vectors

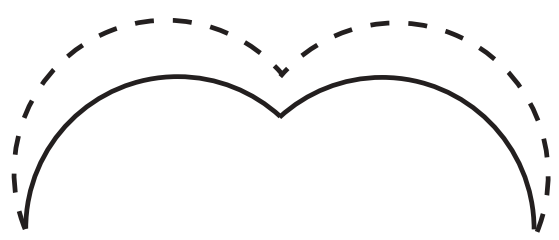

Fig. 2 Double circular arches with same curvature 
conditions for curvatures are very complicated for surface optimization. Therefore, we present a unified approach by using a single Bézier curve to generating optimal curves with different numbers of arches.

The center of curvature $\mathbf{c}(t)$ is defined for $\kappa(t) \neq 0$ as

$\mathbf{c}(t)=\mathbf{x}(t)+\frac{1}{\kappa(t)} \mathbf{n}(t)$

The curve $\mathbf{x}(t)$ is defined as follows by the Bézier curve of order $n$ (Farin 1992):

$\mathbf{x}(t)=\sum_{i=0}^{n} \mathbf{R}_{i} B_{i}^{n}(t)$

where $\mathbf{R}_{i}=\left(R_{i}^{x}, R_{i}^{y}\right)$ and $B_{i}^{n}(t)(i=0, \ldots, n)$ are the control points and the Bernstein polynomials of order $n$, respectively.

In the following examples, $n=5$ and the control polygon is as illustrated in thin lines in Fig. 3. The $(X, Y)$ coordinates are also defined as shown in Fig. 3. Optimal shapes are found from two different initial curves (a) and (b) as shown in Figs. 3 and 4, respectively, where curve (a) has negative curvature, and curve (b) has regions with positive and negative curvatures. Based on the symmetry property, the curve is defined by $\mathbf{R}_{0}, \mathbf{R}_{1}$ and $\mathbf{R}_{2}$. The vector consisting of all the variables is denoted by $\mathbf{R}$.

Let $\mathbf{c}_{0}$ denote the specified center of curvature. Differentiation with respect to $t$ is indicated by a dot. The length of the tangent vector $\dot{\mathbf{x}}(t)$ is denoted by $g(t)$; i.e., $\mathrm{d} s=g(t) \mathrm{d} t$ for the arc-length parameter $s$. Let $(\cdot ; \mathbf{R})$ indicate a function that depends on $\mathbf{R}$. The square of distance between $\mathbf{c}(t ; \mathbf{R})$ and $\mathbf{c}_{0}$ is defined as

$d(t ; \mathbf{R})=\left\|\mathbf{c}(t ; \mathbf{R})-\mathbf{c}_{0}\right\|^{2}$

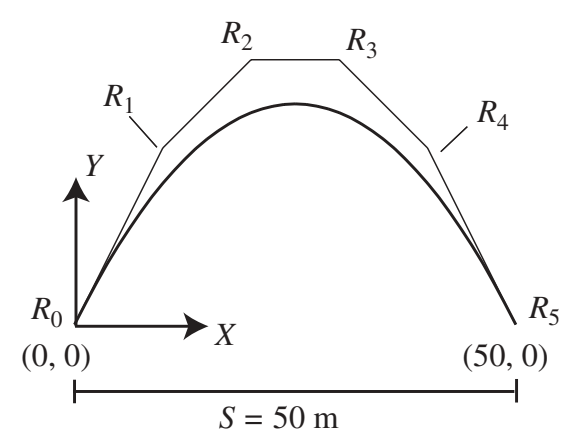

Fig. 3 Initial curve (a)

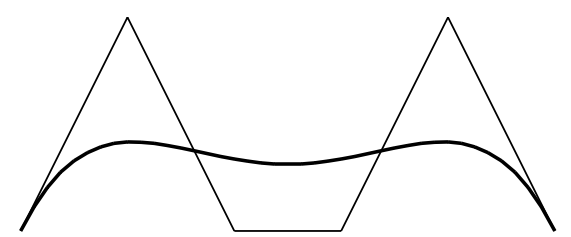

Fig. 4 Initial curve (b) where $\|\cdot\|$ is the Euclidean norm of a vector. The optimization problem may be simply formulated as

P1: Minimize $\int_{0}^{1} d(t ; \mathbf{R}) g(t ; \mathbf{R}) \mathrm{d} t$

An optimal shape as illustrated in Fig. 5 can be obtained by solving P1 from the initial curve (a). Details of the mathematical formulations will be shown in the examples. From the initial curve (b), however, the curve in Fig. 5 has to be reached through an intermediate curve as illustrated in Fig. 6 of which the center of curvature around the center $t=0.5$ is far from $\mathbf{c}_{0}$ and even in the opposite side of the curve. Therefore, the optimal solution of Fig. 5 cannot be obtained by solving P1 from the initial curve (b).

Let $T$ denote the region of $t$ where $\kappa(t)<0$ is satisfied. It is possible that the integration of (4) is to be done only over the region $T$. In this case, however, there is no region for integration if $\kappa(t)>0$ is satisfied throughout the region. Therefore, a curve with positive curvature in $t \in[0,1]$ is obtained from the initial curve (b) because $\mathrm{P} 1$ is a minimization problem with nonnegative objective function and a solution with $\kappa(t)>0$ throughout the region has vanishing objective value that leads to an obvious and meaningless optimal solution. The problem may alternatively be written as

P2: Maximize $\int_{t \in T} \frac{1}{d(t ; \mathbf{R})} g(t ; \mathbf{R}) \mathrm{d} t$

In the process of solving $\mathrm{P} 2$, the design variables are to be modified so that $d(t ; \mathbf{R})$ is reduced and $\kappa(t)<0$ is to be satisfied in wider region of $t$. Therefore, as shown

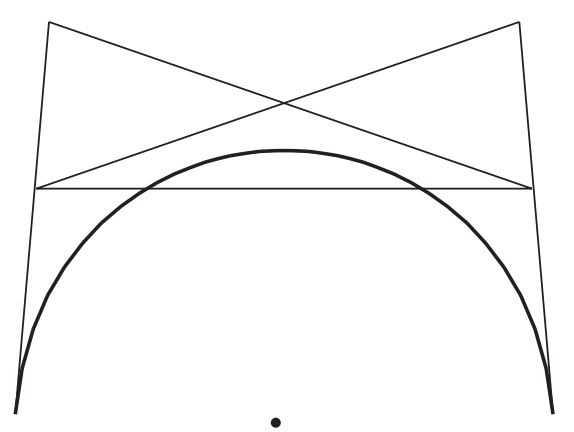

Center of curvature $\boldsymbol{c}_{0}$

Fig. 5 Optimal curve for $\mathbf{c}_{0}=(25,0)$

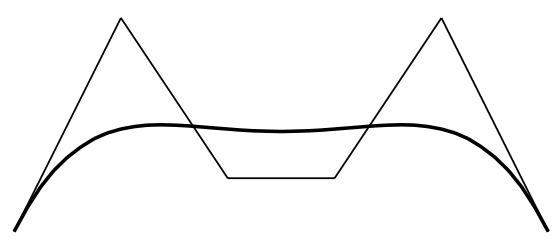

Fig. 6 Intermediate curve during optimization 
in the following examples, the optimal curve in Fig. 5 is successfully obtained from the initial curve (b). Note that the objective function diverges if $d(t ; \mathbf{R})=0$ is satisfied at a point. In order to prevent the divergence, the value of $1 / d(t ; \mathbf{R})$ is replaced by $\bar{d}$ if $1 / d(t ; \mathbf{R})>\bar{d}$, and P2 is reformulated as

P3: Maximize $\int_{t \in T} \min \left\{\frac{1}{d(t ; \mathbf{R})}, \bar{d}\right\} g(t ; \mathbf{R}) \mathrm{d} t$

In numerical implementation, the parameter region is divided uniformly by the interval $\Delta t$, and the value of $t$ at the center of the $i$ th region is denoted by $t_{i}$. Upper and lower bounds for $\mathbf{R}$ are given as $\mathbf{R}^{U}$ and $\mathbf{R}^{L}$, respectively. Finally, the optimization problem to be solved is formulated as

$$
\begin{aligned}
\text { P4: Maximize } & \sum_{t_{i} \in T} \min \left\{\frac{1}{d(t ; \mathbf{R})}, \bar{d}\right\} g\left(t_{i} ; \mathbf{R}\right) \Delta t \\
\text { subject to: } & \mathbf{R}^{L} \leq \mathbf{R} \leq \mathbf{R}^{U} \\
& \mathbf{H}(\mathbf{R}) \leq \mathbf{0}
\end{aligned}
$$

where $\mathbf{H}(\mathbf{R}) \leq \mathbf{0}$ denotes the geometrical constraints given if necessary. The variations of the objective values of $\mathrm{P} 1$ and $\mathrm{P} 4$ between the initial and the optimal solutions are compared in the examples.

A round shape is generated by solving $\mathrm{P} 4$. The mechanically optimal shape, however, is quite different from a round shape. Therefore, we next consider the trade-off between roundness and mechanical property defined by compliance (external work) against static loads. The arch is divided into regions with equal parameter length which are modeled by standard beam elements. Let $\mathbf{F}$ and $\mathbf{U}$ denote, respectively, the vectors of nodal loads and nodal displacements obtained by solving

$\mathbf{K U}=\mathbf{F}$

where $\mathbf{K}$ is the linear elastic stiffness matrix, and dependence of all the variables on $\mathbf{R}$ is assumed. The compliance $W$ is defined by $\mathbf{F}^{T} \mathbf{U}$. The specified structural volume is denoted by $\bar{V}$. The cross-sectional area $A$ is then given by $A=\bar{V} / L$ where $L$ is the total length of the arch. The optimization problem is formulated as

P5: Minimize $W(\mathbf{R})$

$$
\begin{gathered}
\text { subject to: } \mathbf{R}^{L} \leq \mathbf{R} \leq \mathbf{R}^{U} \\
\mathbf{H}(\mathbf{R}) \leq \mathbf{0}
\end{gathered}
$$

Since the roundness and the elastic stiffness defined by using the compliance can be conceived as conflicting performance measures, a multiobjective optimization problem can be formulated for optimizing the two objectives (Cohon 1978). There are many approaches to obtaining all the possible Pareto optimal solutions or to selecting the most preferred solution among the set of Pareto optimal solutions. In this paper, a so called constraint method is used (Cohon 1978). Let $\bar{W}$ denote the specified upper bound for $W$, and consider the following problem:

$$
\begin{aligned}
\text { P6: Maximize } & \sum_{t_{i} \in T} \min \left\{\frac{1}{d(t ; \mathbf{R})}, \bar{d}\right\} g\left(t_{i} ; \mathbf{R}\right) \Delta t \\
\text { subject to: } & W(\mathbf{R}) \leq \bar{W} \\
& \mathbf{R}^{L} \leq \mathbf{R} \leq \mathbf{R}^{U} \\
& \mathbf{H}(\mathbf{R}) \leq \mathbf{0}
\end{aligned}
$$

Note that $\bar{W}$ is given in view of the values of $W$ of the optimal solutions of P4 and P5. A set of Pareto optimal solutions can be generated by solving $\mathrm{P} 6$ for various values of $\bar{W}$.

\section{2 \\ Examples of curve optimization}

Optimal curves are found from the initial shapes (a) and (b), where the span length $S$ is $50 \mathrm{~m}$. The curve is symmetric with respect to the line defined by $Y=25 \mathrm{~m}$. The $(X, Y)$-coordinates of the supports, which are fixed during optimization, are defined as shown in Fig. 3. In the following, the unit of the length is $m$, which is omitted for brevity. The coordinates of $\mathbf{R}_{0}, \mathbf{R}_{1}, \mathbf{R}_{2}$ are $(0,0)$, $(10,20),(20,30)$, respectively, for initial curve (a), and $(0,0),(10,20),(20,0)$ for initial curve (b). The variables are $(X, Y)$-coordinates of $\mathbf{R}_{1}$ and $\mathbf{R}_{2}$, and the number of variables is four. The upper and lower bounds are 50 and -10 , respectively, for the $X$-coordinates, and 50 and -20 for the $Y$-coordinates. Optimization is carried out by IDESIGN 3.5 (Arora and Tseng 1987), and the sequential quadratic programming method is used.

A constraint is given such that the $X$-component of the tangent vector $\dot{\mathbf{x}}(t)$ is nonnegative at the center $t=0.5$. The constraint is explicitly written as

$R_{0}^{x}+3 R_{1}^{x}+2 R_{2}^{x} \leq 3 S$

Note that $Y$-component of $\dot{\mathbf{x}}(t)$ vanishes at $t=0.5$ due to the symmetry condition. Therefore, $\|\dot{\mathbf{x}}(t)\|=0$ and a cusp can exist at the center if (18) is satisfied in equality.

The optimal shape in Fig. 5 has been successfully found from the initial curve (b) by solving P4 with $\mathbf{c}_{0}=(25,0)$, where $\bar{d}=1.0$ and $\Delta t=0.01$. It may be observed from this result that the optimal shape with $\kappa(t)<0$ throughout the region can be found from an initial solution with convex and concave regions.

Let $\mathbf{R}^{\mathrm{I}}$ and $\tilde{\mathbf{R}}$ denote the control points corresponding to the initial curve (b) and the optimal curve, respectively. In order to discuss the efficiency of using the formulation of P4, the intermediate solutions $\hat{\mathbf{R}}$ between $\mathbf{R}^{\mathrm{I}}$ and $\tilde{\mathbf{R}}$ are linearly defined by a parameter $\alpha$ as

$\hat{\mathbf{R}}=\alpha \tilde{\mathbf{R}}+(1-\alpha) \mathbf{R}^{\mathrm{I}}$ 
Let $\beta_{1}$ and $\beta_{4}$ denote, respectively, the objective functions of $\mathrm{P} 1$ and $\mathrm{P} 4$. Variation of $1 / \beta_{4}$ with respect to $\alpha$ is plotted in Fig. 7. It is seen from Fig. 7 that $1 / \beta_{4}$ decreases; i.e. $\beta_{4}$ increases; as the curve approaches the optimal curve. Therefore, the optimal solution can be obtained by solving $\mathrm{P} 4$. On the contrary, $\beta_{1}$ is a discontinuous function of $\alpha$ as shown in Fig. 8. Although the objective value has the minimum value at $\alpha=1$, it is very difficult to reach the optimal solution from the initial solution (b).

If $\mathbf{c}_{0}=(12.5,0)$ is given for the left side with $0 \leq t$ $\leq 0.5$, the optimal curve as shown in Fig. 9 has been reached from the initial curve (a). It may be observed from these results that optimal solutions with various types of curvature distributions can be obtained by specifying the center of curvature, and curves with single and double arches can be obtained without any modification of problem formulation or geometrical modeling.

Next, we consider compliance as a mechanical performance measure. The material is steel where the elastic modulus $E$ is $200.0 \mathrm{GPa}$ and the weight density is $80.0 \mathrm{kN} / \mathrm{m}^{3}$. The cross-section of the arch is sandwich, and the distance between the two flanges is denoted by $h$.

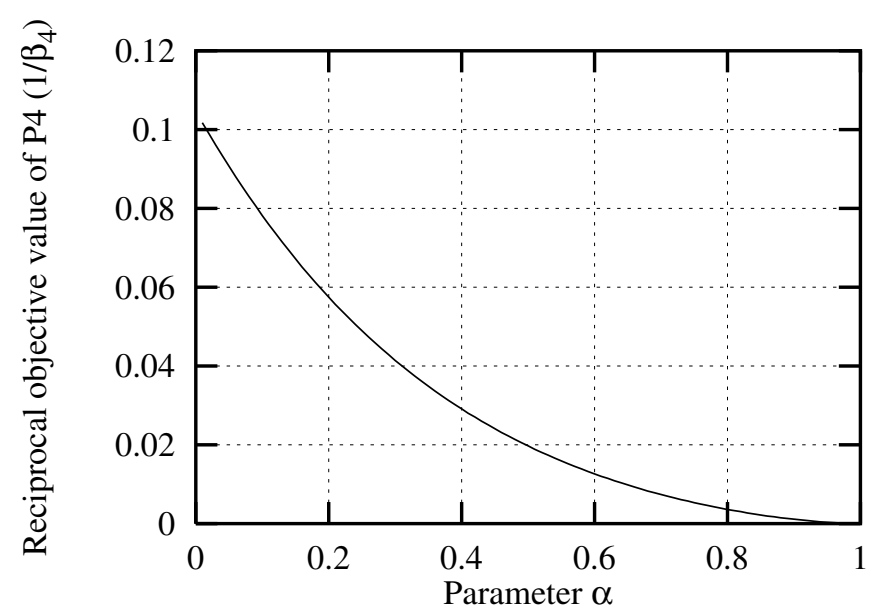

Fig. 7 Variation of the reciprocal objective value of $\mathrm{P} 4$ with respect to $\alpha$

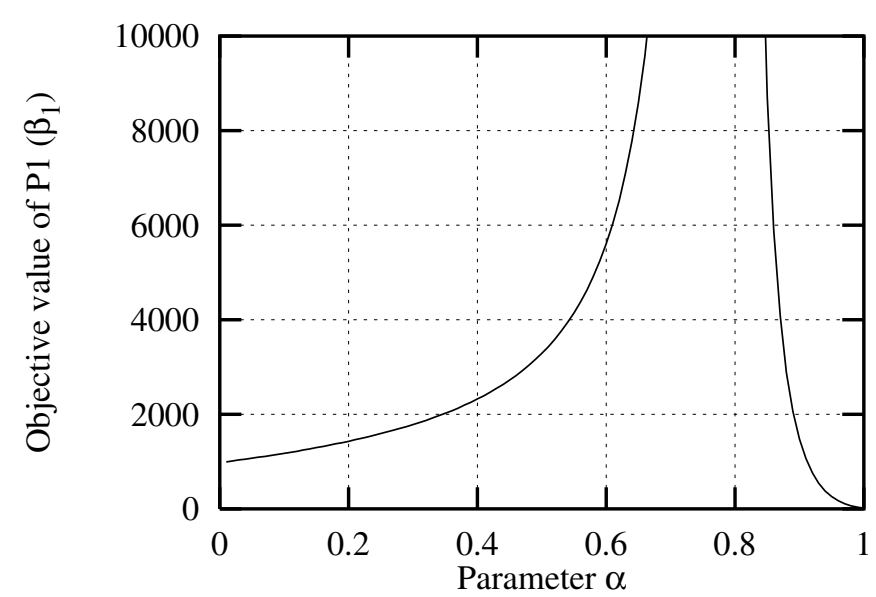

Fig. 8 Variation of the objective value of $\mathrm{P} 1$ with respect to $\alpha$

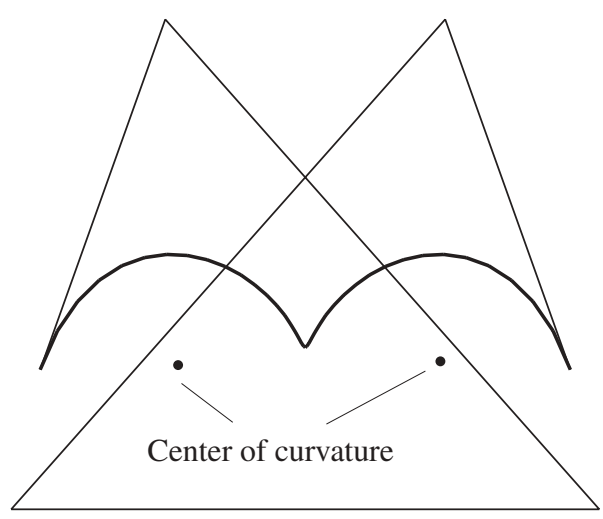

Fig. 9 Optimal shape for $\mathbf{c}_{0}=(12.5,0)$

In this case, the extensional stiffness is $E A$ and the bending stiffness is $E A h^{2} / 4$, where $h=1 \mathrm{~m}$ in the following. The arch has pin supports, and is divided into 20 beam elements. Distributed load of $2.0 \mathrm{kN}$ per unit arclength is applied in the negative $Y$-direction in addition to the self weight. The specified total structural volume $\bar{V}$ is $20.0 \mathrm{~m}^{3}$. The optimal solution for minimizing $W$ is as shown in Fig. 10, where the optimal value of $W$ is $9.3117 \times 10^{2} \mathrm{kNm}$.

It is observed from Figs. 5 and 10 that the optimal shape for minimizing the compliance is quite different from the round shape. The values of $W$ for the optimal round curves in Figs. 5 and 9 are $7.55033 \mathrm{kNm}$ and $9.8876 \times 10^{4} \mathrm{kNm}$, respectively. Problem $\mathrm{P} 6$ has been next solved with $\mathbf{c}_{0}=(25.0,0)$ and $\bar{W}=1.03 \mathrm{kNm}$. Figure 11 shows the obtained optimal shape. It is seen from Fig. 11 that an intermediate solution between Figs. 5 and 10 has been obtained by considering the trade-off between roundness and elastic stiffness.

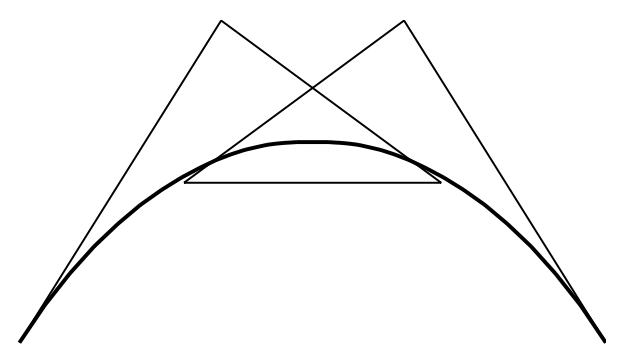

Fig. 10 Optimal solution for minimizing compliance $(W=$ $\left.7.5503 \times 10^{3} \mathrm{kNm}\right)$

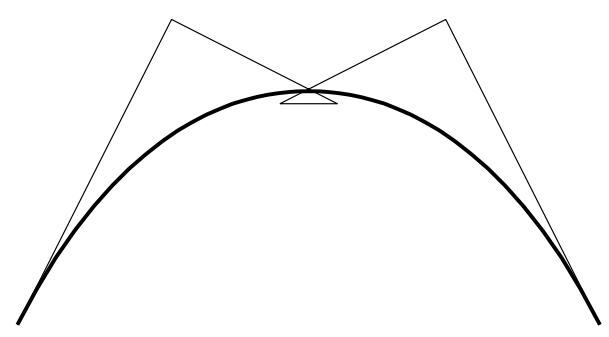

Fig. 11 Optimal solution for $\mathbf{c}_{0}=(25,0)$ under constraint on compliance $\left(W=1.0 \times 10^{4} \mathrm{kNm}\right)$ 


\section{3}

\section{Shape optimization of surfaces}

\section{1}

\section{Problem formulation}

Consider a surface defined by parameters $u$ and $v$ as $\mathbf{X}(u, v)$. The fundamentals on geometry of surfaces may be referred to; e.g., Faux and Pratt (1979), do Carmo (1976). We use curvatures and unit normal vectors also for optimization of surfaces. The unit normal vector of the surface is denoted by $\mathbf{N}(u, v)$. A line in $u$ - or $v$-direction in the parameter space corresponds to a curve in the physical space which is called an isoparametric curve. Let $\mathbf{r}^{u}(u, v)$ and $\mathbf{r}^{v}(u, v)$ denote the unit tangent vectors of the isoparametric curves in $u$ - and $v$-directions, respectively. $\mathbf{N}(u, v)=\mathbf{r}^{u}(u, v) \times \mathbf{r}^{v}(u, v)$ is defined as shown in Fig. 12.

Let $\kappa_{i}(u, v)(i=1,2)$ denote the two principal curvatures at a point on a surface. The centers of curvatures can be defined as

$\mathbf{P}_{i}(u, v)=\mathbf{X}(u, v)+\frac{1}{\kappa_{i}(u, v)} \mathbf{N}(u, v), \quad(i=1,2)$

The formulation (20), however, can not be conveniently used for specifying the desired shape, because two centers of curvatures exist at a point on the surface.

If the Gaussian curvature $K(u, v)=\kappa_{1}(u, v) \kappa_{2}(u, v)$ is used, the center of curvature can be uniquely determined as follows for $K(u, v)>0$ (Hangen et al. 1995):

$\mathbf{C}(u, v)=\mathbf{X}(u, v)+\frac{1}{\sqrt{K(u, v)}} \mathbf{N}(u, v)$

Note that the surface of Fig. 12 has two negative principal curvatures and $K(u, v)>0$.

The surface $\mathbf{X}(u, v)$ is defined by the tensor product Bézier surface as (Farin 1992)

$\mathbf{X}(u, v)=\sum_{i=0}^{n} \sum_{j=0}^{m} \mathbf{R}_{i, j} B_{i}^{n}(u) B_{j}^{m}(v)$

where $\mathbf{R}_{i, j}=\left(R_{i, j}^{x}, R_{i, j}^{y}\right)(i=0, \ldots, n ; j=0, \ldots, m)$ are the control points, and $n=m=5$ in the following examples.

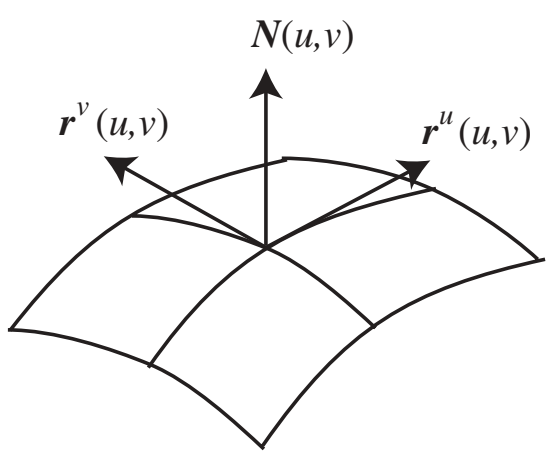

Fig. 12 Unit tangent and normal vectors of a surface
Let $\mathbf{C}_{0}$ denote the specified center of curvature for the definition (21). The parameters $u$ and $v \in[0,1]$ are divided into regions with uniform interval by $\Delta u$ and $\Delta v$, respectively, and the parameter values at the centers of the regions are denoted by $u_{i}$ and $v_{j}$. The determinant of the first fundamental matrix of the surface is denoted by $G(u, v)$. Note that $\mathbf{N}(u, v)$ defined in Fig. 12 is in the same direction irrespective of the sign of the principal curvatures. Let $U$ denote the region where both of the principal curvatures are negative. The square of distance between $\mathbf{C}\left(u_{i}, v_{j} ; \mathbf{R}\right)$ and $\mathbf{C}_{0}$ is defined as

$D\left(u_{i}, v_{j} ; \mathbf{R}\right)=\left\|\mathbf{C}\left(u_{i}, v_{j} ; \mathbf{R}\right)-\mathbf{C}_{0}\right\|^{2}$

The optimization problem for specified center of curvature is formulated as

$$
\begin{array}{ll}
\text { P7: Maximize } & \sum_{\left(u_{i}, v_{j}\right) \in U} \min \left\{\frac{1}{D\left(u_{i}, v_{j} ; \mathbf{R}\right)}, \bar{d}\right\} \times \\
& \sqrt{G\left(u_{i}, v_{j} ; \mathbf{R}\right)} \Delta u \Delta v \\
\text { subject to: } & \mathbf{R}^{L} \leq \mathbf{R} \leq \mathbf{R}^{U} \\
& \mathbf{H}(\mathbf{R}) \leq \mathbf{0}
\end{array}
$$

A round surface is obtained by solving P7.

The center of curvature can alternatively be defined by the isoparametric curves for generating a ribbed shell. Let $\kappa^{u}(u ; v)$ denote the curvature of the isoparametric curve in $u$-direction, where the argument $(u ; v)$ indicates that the parameter is $u$, but the curve is defined for each specified value of $v$. The unit normal vector is denoted by $\mathbf{n}^{u}(u ; v)$. Note that $\kappa^{u}(u ; v)$ of a curve in three dimensional space always has nonnegative value. The center of curvature of the isoparametric curve is given for the region $\kappa^{u}(u ; v) \neq 0$ as

$\mathbf{c}^{u}(u ; v)=\mathbf{X}(u, v)+\frac{1}{\kappa^{u}(u ; v)} \mathbf{n}^{u}(u ; v)$

$\mathbf{c}^{v}(u ; v)$ can be defined similarly.

\section{2}

\section{Examples of surface optimization}

Optimal shapes have been found from the initial shapes (a) and (b) as shown in Figs. 13 and 14, respectively. We only consider the surfaces that are symmetric with respect to the planes defined by $X=25$ and $Y=25$. The $(X, Y, Z)$-coordinates are defined as shown in Fig. 13. Based on the symmetry conditions, the surface is defined by nine control points $\mathbf{R}_{i, j},(i=0,1,2 ; j=0,1,2)$. For the initial shape (a), $\mathbf{R}_{0,0}=(0,0,0), \mathbf{R}_{0,1}=(0,10,0)$, $\mathbf{R}_{0,2}=(0,20,0), \quad \mathbf{R}_{1,0}=(10,0,0), \quad \mathbf{R}_{1,1}=(10,10,20)$, $\mathbf{R}_{1,2}=(10,20,20), \mathbf{R}_{2,0}=(20,0,0), \mathbf{R}_{2,1}=(20,10,20)$, $\mathbf{R}_{2,2}=(20,20,20)$. For the initial shape (b), $\mathbf{R}_{2,2}=$ $(20,20,-10)$ and the remaining control points are same 
as those of (a). The control polygons are plotted in thin lines in Figs. 13 and 14.

The three components of $\mathbf{R}_{0,0}$ are fixed during the optimization process. The control points along the boundary can move only in the vertical planes in which the boundary curves are located. The upper and lower bounds are 50 and 0 , respectively, for $X, Y$-coordinates, and 60 and -20 for $Z$-coordinates. The parameters are divided by $\Delta u=\Delta v=0.0125$. The upper bound $\bar{d}$ in (24) is 1.0. Geometrical constraints are given so that the $X$ - and $Y$-components of the tangent vectors of the isoparametric curves in $u$ - and $v$-directions, respectively, have nonnegative values at the points on the planes of symmetry defined by $u=0.5$ and $v=0.5$. These constraints are then written explicitly as

$R_{0, j}^{x}+3 R_{1, j}^{x}+2 R_{2, j}^{x} \leq 3 S^{x}, \quad(j=0,1,2)$

$R_{i, 0}^{x}+3 R_{i, 1}^{x}+2 R_{i, 2}^{x} \leq 3 S^{y}, \quad(i=0,1,2)$

where $S^{x}$ and $S^{y}$ are the span lengths in $X$ - and $Y$-directions, respectively, which are equal to $50.0 \mathrm{~m}$.

The optimal solution for $\mathbf{C}_{0}=(25,25,0)$ is as shown in Fig. 15 which has been obtained from the initial solution (b) as shown in Fig. 14. A round surface has been successfully reached from a partially concave initial shape. Note that there exist concave regions at four corners. Roundness in almost all the domain, however, has been increased by sacrificing smoothness at the corners of the optimal shape.

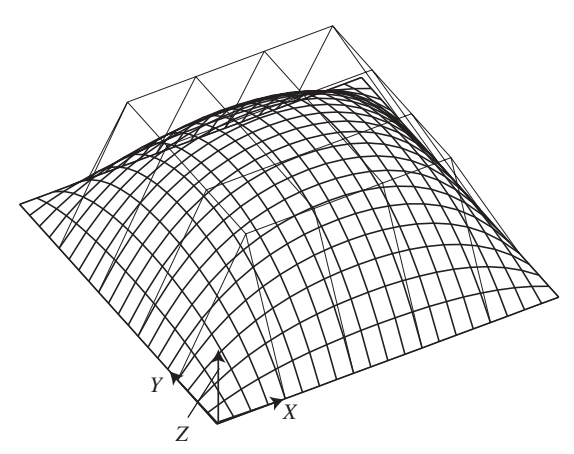

Fig. 13 Initial solution (a)

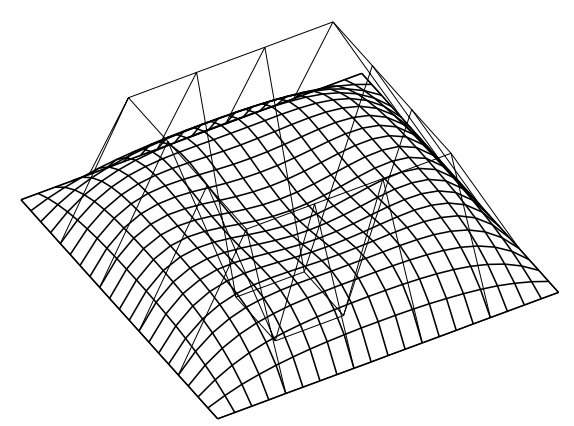

Fig. 14 Initial solution (b)

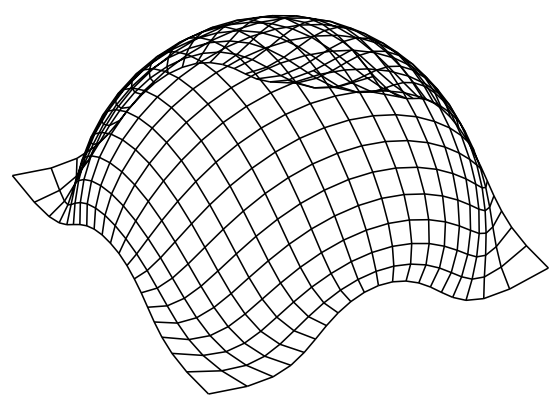

Fig. 15 Optimal solution for $\mathbf{C}_{0}=(25,25,0)$

An optimal shape of Fig. 16 has been found for $\mathbf{C}_{0}=$ $(12.5,12.5,0)$ from the initial shape (a); i.e., a ribbed shell with discontinuity in the tangent vector can be found from a convex initial shape. Therefore, optimal shape with various curvature distributions can be generated by solving P7 from different types of initial shapes.

Consider next a problem of minimizing the compliance under static loads. The curved shell is assumed to be sufficiently thin so that only membrane stresses should be considered. The standard nine-degree-of-freedom triangular element with uniform stresses and strains is used (Zienkiewicz and Taylor 1989). The parameter space $(u, v)$ is divided into $20 \times 20$ regions with same interval. The shell is subjected to distributed load $100.0 \mathrm{~N}$ in negative $Z$-direction per unit area of the surface. The material is steel, and all the displacement components including rotations are fixed along the boundary. The specified structural volume is $10.0 \mathrm{~m}^{3}$.

The optimal solution is as shown in Fig. 17, where the compliance is $5.6934 \times 10^{30} \mathrm{kNm}$. It is seen from Fig. 17 that the optimal shell has a kind of cylindrical shell with parabolic cross-section in each direction of $X$ and $Y$. The value of $W$ for the shells in Figs. 15 and 16 are $6.2064 \times 10^{4} \mathrm{kNm}$ and $5.9848 \times 10^{4} \mathrm{kNm}$, respectively. The trade-off solution for $\mathbf{C}_{0}=(25,25,0)$ and $\bar{W}=$ $7.0 \times 10^{3} \mathrm{kNm}$ is as shown in Fig. 18 which is between the shapes in Figs. 15 and 17.

Finally, a ribbed shell is generated by using the center of curvature of the isoparametric curve given by (27). The inverse of the square of the distance between $\mathbf{c}^{u}(u ; v)$ and the line defined by $X=12.5, Z=0$ for the region $u \in[0,0.5]$ and $v \in[0,1]$ has been minimized to obtain

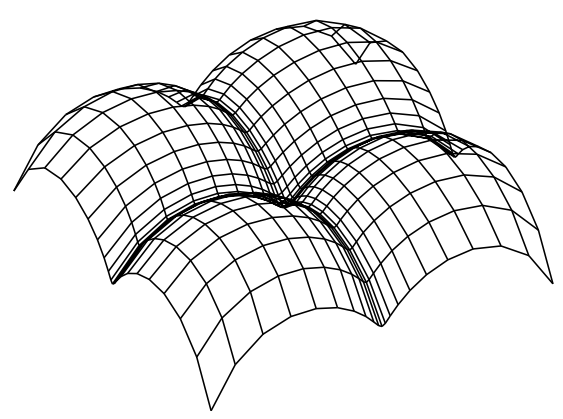

Fig. 16 Optimal solution for $\mathbf{C}_{0}=(12.5,12.5,0)$ 


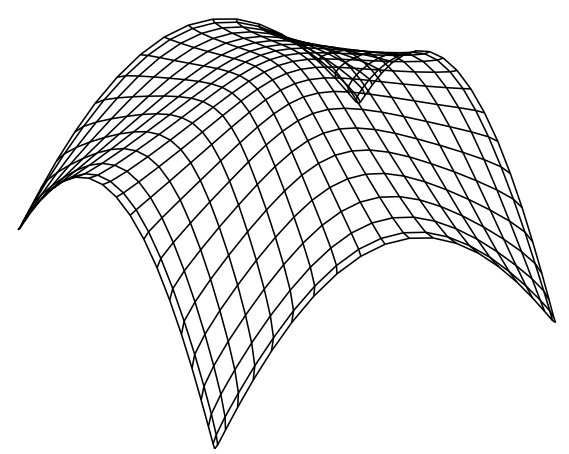

Fig. 17 Optimal shape for minimizing compliance $(W=$ $5.6934 \times 10^{3} \mathrm{kNm}$ )

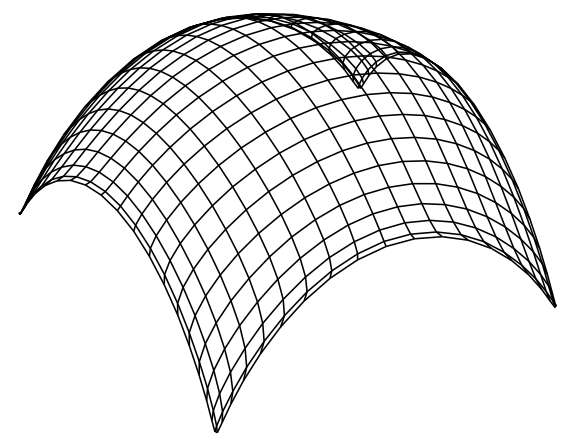

Fig. 18 Optimal shape under compliance constraint $(W=$ $7.0 \times 10^{3} \mathrm{kNm}$ )

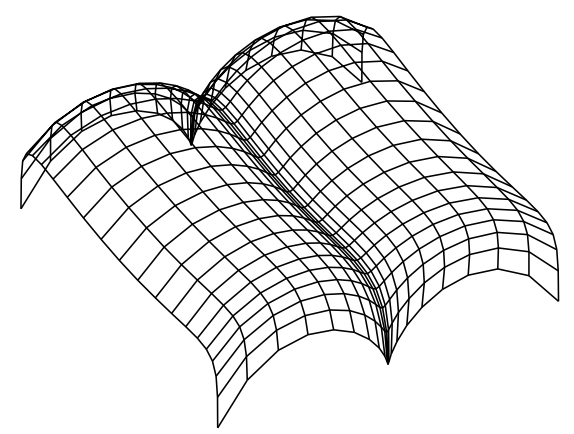

Fig. 19 Optimal shape for specified center of curvature of isoparametric curve

the optimal shape in Fig. 19, where $\bar{d}=1.0$ has also been used, and the integration has been carried out only for the region with $\kappa^{u}(u ; v)<0$. It is observed from Fig. 19 that a ribbed shell can be generated by specifying the center of curvature of the isoparametric curve without any modification of modeling method of the surface.

\section{4}

\section{Conclusions}

A unified approach has been presented for generating round shells with and without ribs from initial shapes with various distributions of curvature. The conclusions drawn from this study are summarized as follows:
1. A smooth convergence of the objective function to the optimal objective value has been demonstrated in the example of a curve optimization by using the objective function defined by the inverse of distance of the center of curvature from the specified point, where the region of the integration of the objective function is restricted by the signs of principal curvatures.

2. Dependence of the optimization result on the initial shape can be successfully avoided by using the proposed formulation, and round curves and surfaces with different numbers of arches and ribs can be generated by specifying the center of curvature without any modification of problem formulation or modeling method.

3. The shape of a ribbed shell or a double arch cannot uniquely be defined only by the curvature distribution and the boundary conditions.

4. The optimal shape for minimizing compliance under constraint on structural volume has been shown to be a doubly curved shell that consists of cylindrical shell in two directions.

5. The constraint approach can be successfully used for obtaining a trade-off design between round and mechanically efficient shapes.

6. A ribbed cylindrical shell can be generated by specifying the distribution of the center of curvature of the isoparametric curves.

It may be observed from these results that the center of curvature is an intrinsic property that directly corresponds to the shape of the curves and surfaces, and various round shapes with and without ribs can be generated by specifying the center of curvatures within a unified problem formulation.

\section{References}

Arora, J.; Tseng, C. 1987: Idesign user's manual, ver. 3.5. Technical report, Optimal Design Laboratory, The University of Iowa

Barnhill, R.E. (ed.) 1994: Geometry Processing for Design and Manufacturing. SIAM

Bletzinger, K.-U. 1999: Structural optimization and form finding of lightweight structures. In Proc. 3rd World Congress of Structural and Multidisciplinary Optimization (WCSMO3)

Cohon, J.L. 1978: Multiobjective Programming and Planning. Mathematics in Science and Engineering 140, Academic Press

do Carmo, M.P. 1976: Differential Geometry of Curves and Surfaces. Prentice-Hall

Du, W.-H.; Schmitt, F.J.M. 1990: On the $g^{1}$ continuity of piecewise Bézier surfaces: a review with new results. Comput. Aided Des. 22(9), 556-573

Farin, G. 1992: Curves and Surfaces for Computer Aided Geometric Design. Academic Press

Faux, I.D.; Pratt, M.J. 1979: Computational Geometry for Design and Manufacture. Ellis Horwood 
Gerostathis, T.P.; Koras, G.D.; Kaklis, P.D. 1999: Numerical experimentation with the Roulier-Rando fairness metrics. Mathematical Engineering in Industry 7(2), 195-210

Greiner, G. 1994: Variational design and fairing of spline surfaces. Computer Graphics Forum 13(3), 143-154

Hangen, H.; Hahmann, S.; Schreiber, T. 1995: Visualization and computation of curvature behaviour of freeform curves and surfaces. Comput. Aided Des. 27(7), 545-552

Kegl, M; Antes, H. 1998: Shape optimal design of elastic space frames with non-linear response. Int. J. Num. Meth. Engng. 43, 93-110

Meier, H.; Nowacki, H. 1987: Interpolating curves with gradual changes in curvature. Comput. Aided Geom. Des. 4, 297-305

Moreton, H.P.; Séquin, C.H. 1992: Functional optimization for fair surface design. Computer Graphics 26(2), 167-176

Moreton, H.P.; Séquin, C.H. 1993: Scale-invariant minimumcost curves: Fair and robust design implements. Computer Graphics Forum 12(3), 473-484

Nowacki, H.; Reese, D. 1983: Design and fairing of ship surfaces. In: Barnhill, R.E.; Bohem, W. (eds.) Surfaces in $C A G D$, pp. 121-134, North-Holland

Nowacki, H.; Liu, D.; Lü, X. 1990: Fairing Bézier curves with constraints. Comput. Aided Geom. Des. 7, 43-55

Ohsaki, M.; Hayashi, M. 2000: Fairness metrics for shape optimization of ribbed shells. J. Int. Assoc. Shells and Spatial Struct. 41(1), 31-39
Ohsaki, M.; Nakamura, T.; Isshiki, Y. 1998: Shape-size optimization of plane trusses with designer's preference. J. Struct. Engng., ASCE 124(11), 1323-1330

Ramm, E. 1992: Shape finding methods of shells. Bulletin of Int. Assoc. for Shell and Spatial Struct 33(2), 89-98

Ramm, E.; Bletzinger, K.-U.; Reitinger, R. 1993: Shape optimization of shell structures. Bulletin of Int. Assoc. for Shell and Spatial Struct 34(2), 103-121

Rando, T.; Roulier, J.A. 1991: Designing faired parametric surfaces. Comput. Aided Des. 23, 492-497

Roulier, J.; Rando, T. 1994: Measures of fairness for curves and surfaces. In Spadis, N.S. (ed.) Designing Fair Curves and Surfaces, pp. 75-122, SIAM

Rozvany, G.I.N. (ed.) 1992: Shape and Layout Optimization of Structural Systems and Optimality Criteria Methods. Springer

Sarraga, R.F. 1998: Recent methods for surface shape optimization. Comput. Aided Geom. Des. 15, 417-436

Subramainan, V.A.; Suchithran, P.R. 1999: Interactive curve fairing and bi-quintic surface generation for ship design. Int. Shipbuild. Progr. 46(446), 189-208

Welch, W.; Witkin, A. 1992: Variational surface modeling. Computer Graphics 26(2), 157-166

Zienkiewicz, O.C.; Taylor, R.L. 1989: The Finite Element Method. McGraw-Hill 